\title{
Physics and detector studies with the very forward calorimeters at a future linear collider
}

\section{Ivanka Bozovic-Jelisavcic [on behalf of the FCAL Collaboration]}

Vinca Institute of Nuclear Sciences, University of Belgrade

M. Petrovica Alasa 12-14 Belgrade, Serbia

E-mail: ibozovicevinca.rs

\begin{abstract}
The very forward region at a future $\mathrm{e}^{+} \mathrm{e}^{-}$collider will be instrumented with calorimeters dedicated primarily for precise measurement of the integral luminosity with the precision required at a percent (CLIC) or at a permille level (ILC). As the constraints on luminosity precision come from the physics programs at future linear colliders, physics and machinerelated systematic effects are discussed as the main sources of uncertainty in luminosity measurement.
\end{abstract}




\section{Introduction}

Integrated luminosity measurement at a future linear collider will be performed by counting Bhabha events reconstructed in the luminometer fiducial volume. To match the physics benchmarks (i.e. W-pair production, fermion pair-production cross-section measurements) that might be of particular interest for the new physics, luminosity should be known at the level of $10^{-3}$ or better at ILC and at a percent level at CLIC. This can be achieved with finely granulated calorimeters of high energy and polar angle resolution. Compact calorimeters with a small Moliere radius of $\sim 1.5 \mathrm{~cm}$ are foreseen in the very forward region. Luminometer for a future linear collider will be designed as a finely segmented sampling calorimeter with tungsten absorber of $1 \mathrm{X}_{0}$ thickness interspersed with the high-ohmic n-type silicon sensors. To reduce systematic biases from the mechanical precision, a laser based monitoring system has been developed for ILC to control the position of the luminometer over short distances within a micron [1]. ILD model [2] for ILC is assumed at a center-of-mass energy of $500 \mathrm{GeV}$ and $1 \mathrm{TeV}$. The study is extended to $3 \mathrm{TeV}$ center-of-mass energy, assuming the design of the very forward region for the CLIC detector [3].

One of the main uncertainties in luminosity measurement at a future $\mathrm{e}^{+} \mathrm{e}^{-}$collider at $\mathrm{TeV}$ energies comes from the effects induced by space charges of the opposite beams. Beamstrahlung and electromagnetic deflection induced by the field of the opposite bunch, together with ISR, result in the change of the four-vectors of the final state particles, consequently causing the deviation of the polar angles and counting losses in the luminometer. The beam induced effects are particularly pronounced at higher energies, rising from $12.8 \%$ at $500 \mathrm{GeV}$ at ILC $[4,5]$ in the upper $20 \%$ of the luminosity spectrum up to $70 \%$ counting loss in the $3 \mathrm{TeV}$ CLIC case for the whole spectrum [6]. Corrective methods [6] developed to take this into account are reviewed in this paper.

\section{Forward region layout}

Two special calorimeters are foreseen to instrument the very forward region down to approximately $5 \mathrm{mrad}(10 \mathrm{mrad})$ at ILC (CLIC): a luminometer designed to measure the rate of the low angle Bhabha scattering and the beam calorimeter that will allow fast luminosity estimate and extraction of the beam parameters. In addition, sensors of the beam calorimeter have to be radiation hard since the large amount of beamstrahlung pairs $(\sim 10 \mathrm{TeV} / \mathrm{BX}$ at ILC) will be deposited in this detector. Both detectors are realized as sampling calorimeters using 1 $\mathrm{X}_{0}$ tungsten plates as absorber. Except for the dedicated measurements, these detectors will serve to improve the overall hermeticity providing at the same time shielding for the inner tracking detectors.

Calorimeters foreseen to instrument the very forward region at CLIC are practically of the same design as for ILC, with the increased number of sensor-absorber layers due to higher energies (40 layers at CLIC versus 30 at ILC), differing only in apertures and distances from the IP optimized to take into account severe background from coherent and incoherent pairs $\left(10^{8}\right.$ and $10^{5}$ pairs produced per bunch crossing, respectively [7]). Angular coverage of the forward region detectors extends from $10 \mathrm{mrad}$ to $110 \mathrm{mrad}$. At ILC and CLIC luminometers cover 31- 
$78 \mathrm{mrad}$ and 38-110 mrad in polar angle, respectively. Layout of the very forward region at ILC and CLIC is illustrated in Figure $1[2,3]$ for the ILD detector concept.
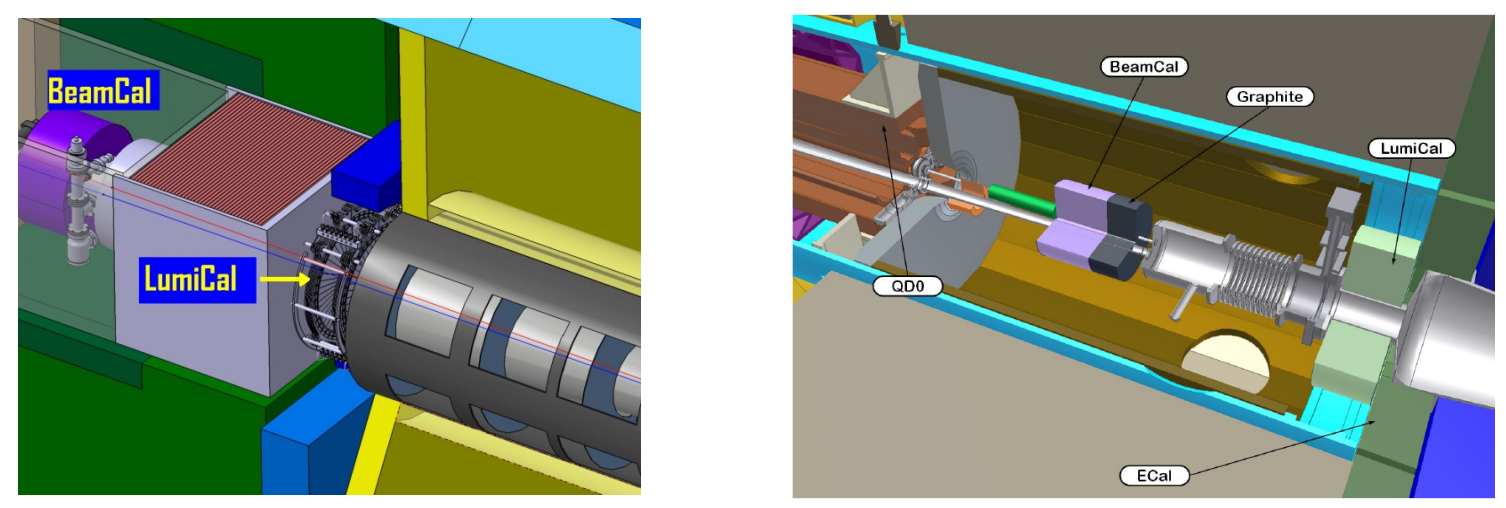

Figure 1: Layout of the very forward region at ILC (left) and CLIC (right).

\section{Method of luminosity measurement}

Luminosity at a future linear collider will be measured by determining the number of Bhabha scattering $(\mathrm{N})$ corresponding to the theoretical cross-section $(\sigma)$ in the detector fiducial volume as given in Eq. 1. This is counting experiment based on the theoretically well described QED process realized via t-channel photon exchange at small angles.

$$
\mathrm{L}=\mathrm{N} / \sigma
$$

However, the number of experimental counts is not exactly $\mathrm{N}$ from Eq. 1 since there are background particles miscounted as a signal and lost counts due to the deviation of polar angles induced by the beam-beam effects. Thus the number of Bhabha events counted experimentally has to be corrected for the estimated miscounts and count losses that we can determine, either experimentally or from simulation, with some intrinsic uncertainty. This uncertainty translates to the systematic uncertainty of the integral luminosity measurement. We propose the method to correct for the beam induced effects in a way that the residual uncertainty of the counting losses is in line with the required luminosity precision, or better. Physics background is taken as a fullsize effect since no uncertainty of the cross-section of these processes is yet known at the NLO level at $\mathrm{TeV}$ energies.

Guinea-Pig V1.4.4 [8] and BHWIDE V4.04[9] are used to simulate approximately $10^{6}$ Bhabha events with the nominal ILC beam parameters. Four-fermion background processes are simulated using WHIZARD V1.2 [10] multi-particle event generator. Detector related effects are simulated either using detector simulation software BARBIE V5.0 at ILC [11] or by the appropriate Gaussian smearing at CLIC.

\section{Beam induced effects}

Initial state electron and positron undergo beamstrahlung in addition emitting ISR while the final state Bhabha particles get scattered by the EM field of the opposite bunch. As mentioned in Section 1, the net effect is a significant counting loss in the detector fiducial volume. The most dominant source of the counting loss comes from the radiation emission 
through beamstrahlung and ISR from the initial state, since the electromagnetic deflection is rather small effect $\left(\sim 10^{-3}\right)$ at $\mathrm{TeV}$ energies.

Center-of-mass frame of the colliding particles, after the beamstrahlung and ISR and prior to FSR (collision frame) is moving w.r.t. the laboratory frame with the velocity $\beta_{\text {coll }}=(\mathrm{v} / \mathrm{c})_{\text {coll }}$ that is calculable from the reconstructed final state polar angles $\theta$ :

$$
\beta_{\text {coll }}=\frac{\sin \left(\theta_{1}^{l a b}+\theta_{2}^{l a b}\right)}{\sin \theta_{1}^{l a b}+\sin \theta_{2}^{l a b}}
$$

As illustrated in Figure 2 [5], events with the larger $\beta_{\text {coll }}$ are more probable to miss the detector fiducial volume. For $\beta_{\text {coll }}$ larger than some critical value $\beta^{*}$ (approximately 0.24 at ILC and 0.3 at CLIC), events are irreducibly lost. However for $\beta_{\text {coll }} \leq \beta^{*}$ counting loss can be corrected for by appropriate weighting $\mathrm{w}\left(\beta_{\text {coll }}\right)$ on the event-by-event basis.

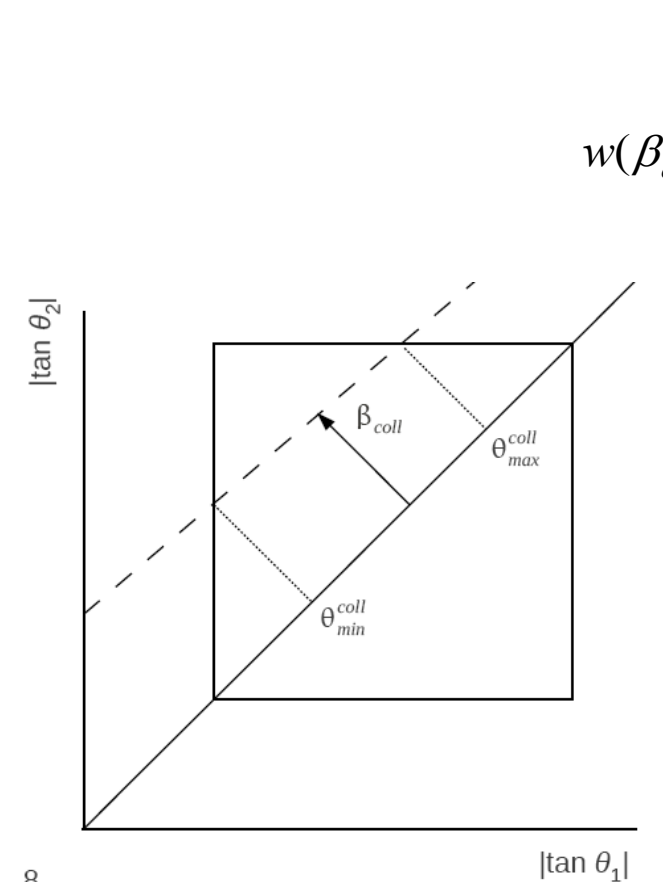

Figure 2: Events with the larger collision frame velocity $\beta_{\text {coll }}$ tend to be lost from the fiducial volume (square).

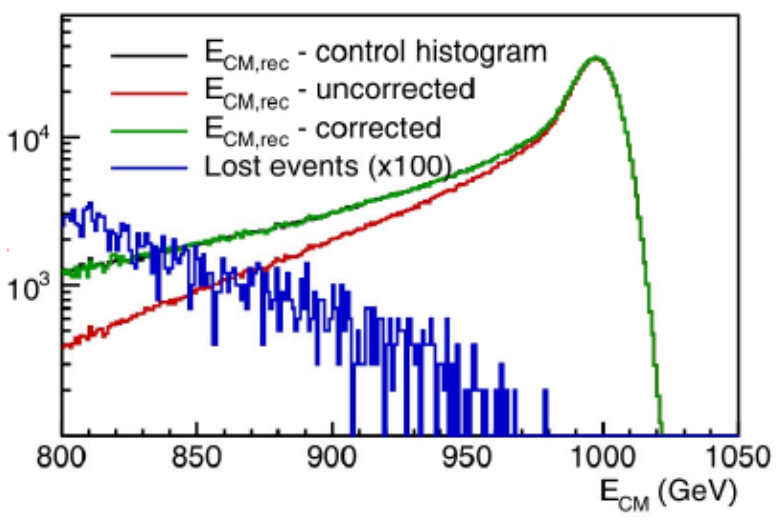

Figure 3: Uncorrected, corrected and lost events above $80 \%$ of the nominal center-ofmass energy at ILC.

\subsection{ILC case}

In order to suppress background from physics processes (see Chapter 5) only events above $80 \%$ of the nominal center-of-mass energy are selected for the luminosity measurement. The fraction of Bhabha events in this energy range that will be lost from the detector fiducial volume due to the high $\beta_{\text {coll }}$ is fairly small $(\sim 1.3 \%)$ at ILC energies. This bias of the method translates to the luminosity uncertainty. It can be further corrected for, however not in the completely simulation independent manner. Figure 3 [4] illustrates uncorrected, corrected and lost counts at $1 \mathrm{TeV}$ ILC. 


\subsection{CLIC case}

Due to higher energy and bunch fields, counting losses due to beamstrahlung are much more pronounced at CLIC leading to the counting losses of 43\% [5] even in the peak of the luminosity spectrum (above $80 \%$ of the nominal center-of-mass energy). After the event weight correction, the residual counting bias is at the level of a few permille ( $\sim 5 \%$ o ) or even below $1 \%$ o in the top 5\% percent of the spectrum [5]. As illustrated in Figure 4 [6], the significant fraction of spectrum is irreducibly lost below $80 \%$ of the nominal center-of-mass energy due to events missing the luminometer fiducial volume because of the deviated polar angles.

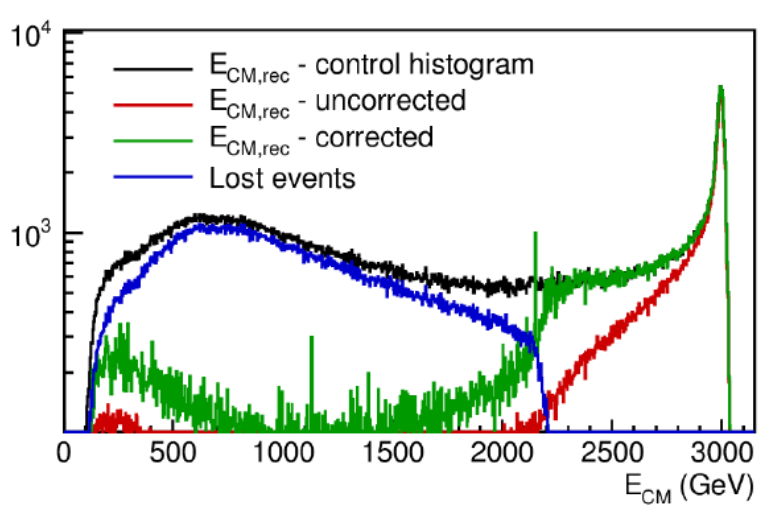

Figure 4: Uncorrected, corrected and lost events at $3 \mathrm{TeV}$ CLIC.

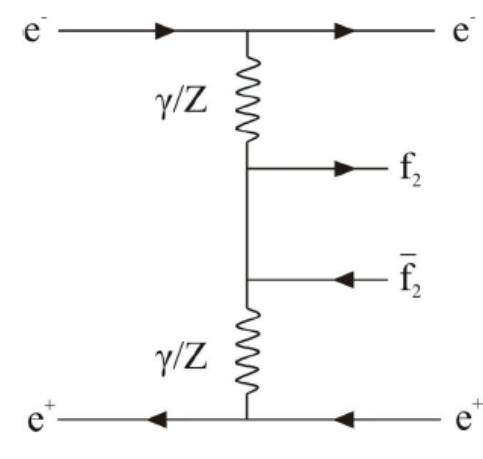

Figure 5: Dominant Feynman graph for physics background production.

\section{Physics background at ILC}

Electron spectators produced in four-fermion processes (Figure 5) can be miscounted as a signal since they are emitted at low polar angles carrying the most of the beam energy. Physics background contamination is at the level of several permille at ILC energies ( $\sim 6$ permille at $500 \mathrm{GeV}$ ) without any selection. If, however, only events above $80 \%$ of the nominal center-ofmass energy with acoplanarity less than $87 \mathrm{mrad}$ are considered for luminosity measurement, physics background is reduced to $\sim 2 \%$ at $500 \mathrm{GeV}$ and below $1 \%$ at $1 \mathrm{TeV}$. With the same selection, the fraction of the lost events due to the beamstrahlung and ISR gets reduced $\leq 0.5 \%$ o [4] since this type of isolation cuts prefer events with the smaller energy losses (smaller $\beta_{\text {coll }}$ ). Taking into account other systematic effects as in [12], we prove that the overall uncertainty of the integral luminosity is $\leq 3 \%$ at ILC energies taking into account simulation dependent corrections and $\leq 5 \%$ if one corrects in a simulation independent manner.

\section{Conclusions}

Following the requirements for precision of luminosity measurement at a future linear collider, physics and machine induced effects are understood at the great level of details. Corrective method has been developed to take into account counting losses due to beamstrahlung and the initial state radiation. The above reduces very large systematic uncertainties of the integral luminosity (of more than ten percent in top $20 \%$ of the luminosity 
spectrum at ILC) to the permille level. Event selection for luminosity measurement at ILC is proposed to comprehend both the beam induced effects and miscounts due to the physics background. It has been proven that the overall uncertainty of the luminosity measurement at ILC is at the level less than a three (five) permille for the simulation dependent (independent) corrections.

\section{References}

[1] J. A. Aguilar et al, Studies on Inner Detectors Alignment in ILD, Proceedings of the XIX Workshop of the Collaboration on Forward Calorimetry (FCAL) at Future Linear Collider, Vinca Institute of Nuclear Sciences, Belgrade, Serbia, 2011, 9-12 pp, ISBN 978-86-7306-114-6.

[2] H. Stoeck, et.al. [ILD concept group], The International Large Detector Letter of Intent, ISSN 0418-9833, ISBN 978-3-935702-42-3.

[3] L. Linssen, A. Miyamoto, M. Stanitzki, H. Weerts (Editors), CLIC Conceptual Design Report: Physics and Detectors at CLIC (Vol.2), September 2011 https://edms.cern.ch/document/1160419

[4] I. Smiljanic [on behalf of the FCAL Collaboration], Correction methods for counting losses induced by the beam-beam effects in luminosity measurement at ILC, International Workshop on Future Linear Colliders (LCWS12), Arlington, Texas, USA, October 2012

http://ilcagenda.linearcollider.org/getFile.py/access?contribId=178 \&session Id=12\&resId=1\&material Id=slides \&conf Id=5468

[5] S. Lukic, I. Smiljanic, Correction of angular counting biases in luminosity measurement at ILC, PREL-LC-PHSM-2012-001.

[6] S. Lukic, Correction of beam-beam effects in luminosity measurement in the forward region at CLIC, LCD-Note-2012-008, CERN, May 2012

https://edms.cern.ch/file/1224621/5/LCD-Note-2012-008.pdf

[7] A. Sailer, D. Dannheim, Beam-Induced Backgrounds in the CLIC Detectors, LCD-Note-2011021, CERN, 2012.

[8] D. Schulte, Study of Electromagnetic and Hadronic Background in the Interaction Region of the TESLA Collider, Ph.D. thesis, Hamburg University, 1996.

[9] S. Jadach, W. Placzek, E. Richter-Was, B. Ward, and Z. Was, Upgrade of the Monte Carlo program BHLUMI for Bhabha scattering at low angles to version 4.04, Computer Physics Communications, vol. 102 p. 229, 1997.

[10] W. Kilian, T. Ohl, J. Reuter, WHIZARD: Simulating Multi Particle Processes et LHC, arXiv:hep-ph/0708.4233.

[11] B. Pawlik, BARBIE 5.0 - Easy to use simulation package of the TESLA LAT detector, IFJ PAN Krakow, Poland.

[12] H. Abramowicz, I. Bozovic-Jelisavcic, T. Jovin, M. Pandurovic, I. Smiljanic et.al. [FCAL Collaboration], Forward Instrumentation for ILC Detectors, JINST 5 P12002, 28 pp., December 2010. 